Canad. Math. Bull. Vol. 56 (4), 2013 pp. 695-708

http://dx.doi.org/10.4153/CMB-2012-034-x

(C) Canadian Mathematical Society 2012

\title{
Carmichael Meets Chebotarev
}

\author{
William D. Banks, Ahmet M. Güloğlu, and Aaron M. Yeager
}

Abstract. For any finite Galois extension $K$ of $\mathbb{Q}$ and any conjugacy class $C$ in $\operatorname{Gal}(K / \mathbb{Q})$, we show that there exist infinitely many Carmichael numbers composed solely of primes for which the associated class of Frobenius automorphisms is $C$. This result implies that for every natural number $n$ there are infinitely many Carmichael numbers of the form $a^{2}+n b^{2}$ with $a, b \in \mathbb{Z}$.

\section{Introduction}

For every prime number $N$, Fermat's little theorem asserts that

$$
a^{N} \equiv a(\bmod N) \quad \text { for all } a \in \mathbb{Z} .
$$

Around 1910, Carmichael began an in-depth study of composite numbers $N$ with this property, which are now known as Carmichael numbers. In 1994 the existence of infinitely many Carmichael numbers was established by Alford, Granville, and Pomerance [1]. The aim of this work is to prove the following extension of their result.

Theorem 1.1 Let $K / \mathbb{Q}$ be a finite Galois extension, and let $C$ be a fixed conjugacy class in $\operatorname{Gal}(K / \mathbb{Q})$. Then there are infinitely many Carmichael numbers that are composed solely of primes for which the associated class of Frobenius automorphisms is the class $C$.

Let $K / \mathbb{Q}$ be an arbitrary number field and $K_{0}$ its Galois closure. Taking the conjugacy class of the identity automorphism of $K_{0}$ in Theorem 1.1, it follows that there exist infinitely many Carmichael numbers composed solely of primes that split completely in $K_{0}$. Since such primes must also split completely in $K$, we deduce the following statement, recovering a recent result of Grantham [8, Theorem 2.1] on the existence of infinitely many Carmichael-Frobenius numbers with respect to $K$.

Corollary 1.2 For any fixed algebraic number field K, there are infinitely many Carmichael numbers that are composed solely of primes that split completely in $K$.

As prime numbers and Carmichael numbers are linked by the common property (1.1), it is natural to ask whether certain questions about primes can also be settled for Carmichael numbers; see [2,3,6]. For example, it is well known that for every natural number $n$, there are infinitely many primes of the form $a^{2}+n b^{2}$ with $a, b \in \mathbb{Z}$ (see the book [4] by Cox), and thus it is natural to ask whether the same result holds for the set of Carmichael numbers. In view of Corollary 1.2, we give an affirmative answer to this question.

Received by the editors November 14, 2011; revised August 16, 2012.

Published electronically September 21, 2012.

AMS subject classification: 11N25, 11R45.

Keywords: Carmichael numbers, Chebotarev density theorem. 
Corollary 1.3 For any fixed integer $n \geqslant 1$, there are infinitely many Carmichael numbers of the form $a^{2}+n b^{2}$ with $a, b \in \mathbb{Z}$.

To see why, let $S_{n}=\left\{a^{2}+n b^{2}: a, b \in \mathbb{Z}\right\}$, and let $K_{n}$ be the ring class field associated with the order $\mathbb{Z}[\sqrt{-n}]$ in the imaginary quadratic field $\mathbb{Q}(\sqrt{-n})$. According to [4, Theorem 9.4], if $p$ is an odd prime not dividing $n$, then $p$ splits completely in $K_{n}$ if and only if $p \in S_{n}$. Applying Corollary 1.2 with $K=K_{n}$, we see that there are infinitely many Carmichael numbers $N$ composed solely of primes $p \in S_{n}$. Since $S_{n}$ is closed under multiplication, every such $N$ also lies in $S_{n}$, and the corollary follows.

In a different direction, taking $K=\mathbb{Q}\left(\mu_{d}\right)$, where $\mu_{d}$ is a primitive $d$-th root of unity, we recover the following result.

Corollary 1.4 For any coprime integers $a$ and $d \geqslant 1$, there are infinitely many Carmichael numbers composed solely of primes $p \equiv a(\bmod d)$.

Matomäki [11] has recently shown that whenever $\operatorname{gcd}(a, m)=1$ and $a$ is a quadratic residue $\bmod m$, there are infinitely many Carmichael numbers in the progression $a$ mod $m$. Assuming the necessary compability between the numbers $a, m$ and the conjugacy class $C$ in $\operatorname{Gal}(K / \mathbb{Q})$, it should be possible to combine the methods of [11] with those in our proof of Theorem 1.1 to show that there are infinitely many Carmichael numbers in the arithmetic progression $a$ mod $m$ that are composed solely of primes for which $C$ is the associated class of Frobenius automorphisms. We thank the referee for posing this question and leave it as an open problem for the interested reader.

\section{Preliminaries}

Let $K / \mathbb{Q}$ be a finite Galois extension of degree $n_{K}=[K: \mathbb{Q}]$ and absolute discriminant $\mathscr{D}_{K}$. We put

$$
\mathbb{N}_{K}=\left\{d \in \mathbb{N}: \operatorname{gcd}\left(d, \mathscr{D}_{K}\right)=1\right\} .
$$

For any Galois extension $M / N$ and any unramified prime ideal $\mathfrak{p}$ of $N$, we denote by $(\mathfrak{p}, M \mid N)$ the conjugacy class of Frobenius automorphisms of $\operatorname{Gal}(M / N)$ corresponding to the prime ideals of $M$ above $\mathfrak{p}$.

Given a conjugacy class $C$ in $\operatorname{Gal}(K / \mathbb{Q})$, let

$$
\mathscr{P}_{C}=\left\{p \in \mathbb{N}_{K}: p \text { prime, }(p, K \mid \mathbb{Q})=C\right\} .
$$

For $d \in \mathbb{N}$ and $M$ a number field, put $M_{d}=M\left(\mu_{d}\right)$, where $\mu_{d}$ is a primitive $d$-th root of unity. According to [15, Proposition 2.7], the discriminant of $\mathbb{Q}_{d}$ is

$$
\mathscr{D}_{\mathbb{Q}_{d}}=(-1)^{\phi(d) / 2} \frac{d^{\phi(d)}}{\prod_{p \mid d} p^{\phi(d) /(p-1)}},
$$

where $\phi(\cdot)$ is the Euler function. 
Lemma 2.1 For each $d \in \mathbb{N}_{K}, K_{d}$ is a Galois extension of $\mathbb{Q}$ of degree $n_{K} \phi(d)$ with discriminant

$$
\mathscr{D}_{K_{d}}=\mathscr{D}_{K}^{\phi(d)} \mathscr{D}_{\mathbb{Q}_{d}}^{n_{K}} .
$$

Furthermore, $\operatorname{Gal}\left(K_{d} / \mathbb{Q}\right) \simeq \operatorname{Gal}(K / \mathbb{Q}) \times \operatorname{Gal}\left(\mathbb{Q}_{d} / \mathbb{Q}\right)$, where the isomorphism is given by the restriction map $\sigma \rightarrow\left(\sigma_{\mid K}, \sigma_{\mid \mathbb{Q}_{d}}\right)$.

Proof In view of (2.1) and (2.2), the discriminants $\mathscr{D}_{K}$ and $\mathscr{D}_{\mathbb{Q}_{d}}$ are coprime for every $d \in \mathbb{N}_{K}$. Put $L=K \cap \mathbb{Q}_{d}$. By [13, Ch. 3, Corollary 2.10], the absolute discriminant $\mathscr{D}_{L}$ of $L$ divides both $\mathscr{D}_{K}$ and $\mathscr{D}_{\mathbb{Q}_{d}}$; thus, $\mathscr{D}_{L}=1$ and $K \cap \mathbb{Q}_{d}=L=\mathbb{Q}$. The result now follows from [13, Ch.1, Proposition 2.11] and [5, 14.4, Proposition 21 and Corollary 22].

The constants $c_{0}, c_{1}, c_{2}, \ldots$, that appear in our proofs are assumed to be positive and depend only on the field $K$. All constants implied by the symbols $O$, $\ll$, and $\gg$ are absolute; we write $O_{K}$, $\ll_{K}$, and $\gg_{K}$ to indicate that the implied constant depends on $K$.

\section{Zeros of Dedekind Zeta Functions}

For each $d \in \mathbb{N}_{K}$, let $\zeta_{d}(s)$ be the Dedekind zeta function $\zeta_{K_{d}}(s)$ associated with the field $K_{d}$ considered in Section 2.

Lemma 3.1 There are constants $c_{1}, c_{2}>0$ depending only on $K$ with the property that for all $T \geqslant 1$ and $U \geqslant 2$ there exists a proper integral ideal $\mathfrak{f}=\mathfrak{f}(K, U, T)$ of $K$ such that for any $d \in \mathbb{N}_{K}$ with $d \leqslant U$, we have $\mathfrak{f} \mid d \mathcal{O}_{K}$, where $\mathcal{O}_{K}$ is the ring of integers of $K$, whenever $\zeta_{d}(s)$ has a zero $\beta+i \gamma$ in the region

$$
\Omega(T, U)=\left\{\beta+i \gamma: \beta \geqslant 1-\frac{c_{1}}{\log \left(c_{2} T U\right)},|\gamma| \leqslant T\right\} .
$$

Proof We use the notation of $[16, \S 1]$. For each $d \in \mathbb{N}_{K}$ with $d \leqslant U$, and any Dirichlet character $\chi$ modulo $(d)=d \mathcal{O}_{K}$ of conductor $\mathfrak{f}_{\chi}$, we see that

$$
d_{\chi}:=\left|\mathscr{D}_{K}\right| \mathrm{N}_{K / \mathbb{Q}}\left(\mathfrak{f}_{\chi}\right) \underset{K}{\ll} d^{n_{K}} \leqslant U^{n_{K}} .
$$

Hence, it follows that $d_{\chi} \leqslant\left(c_{2} U\right)^{n_{K}}$ for some constant $c_{2}=c_{2}(K)$. Applying [16, Theorem 1.9] with $Q=\left(c_{2} U\right)^{n_{K}}$ and $\mathscr{L}=\log \left(Q T^{n_{K}}\right)=n_{K} \log \left(c_{2} T U\right)$, we see that for some constant $c_{1}=c_{1}(K)$, any Hecke $L$-function $L(s, \chi)$ with $d_{\chi} \leqslant Q$ has at most one zero in the region $\Omega(T, U)$. Moreover, the remark following [16, Theorem 1.9] asserts that there is at most one function $L\left(s, \chi_{*}\right)$ vanishing in $\Omega(T, U)$ among all $L(s, \chi)$ associated with primitive characters $\chi$ with $d_{\chi} \leqslant Q$. If such a zero exists, then it is a real number $\beta_{*}$ (which can be bounded in terms of $Q$ ). For such a zero we have

$$
\beta_{*} \geqslant 1-\frac{c_{1}}{\log \left(c_{2} T U\right)} \geqslant 1-\frac{c_{1}}{\log c_{2}} .
$$

Replacing $c_{1}$ by a smaller constant (which also depends only on $K$ ), we can assume that $\zeta_{K}\left(\beta_{*}\right) \neq 0$, i.e., $\chi_{*}$ is not the trivial character. 
By [13, Ch.7, Corollary 10.5],

$$
\zeta_{d}(s)=\zeta_{K}(s) \prod_{\chi \neq 1} L\left(s, \chi, K_{d} \mid K\right)
$$

is the product of Artin $L$-functions, where $\chi$ runs over the irreducible characters of $\operatorname{Gal}\left(K_{d} / K\right)$. Let $K_{\chi}$ be the fixed field of the kernel of $\chi$. Then $\chi$ is injective as a character of $\operatorname{Gal}\left(K_{\chi} / K\right)$. Hence, by [13, Ch.7, Theorem 10.6] there exists a primitive Dirichlet character $\widetilde{\chi}$ modulo the conductor $\mathfrak{f}_{\chi}$ of the extension $K_{\chi} / K$ such that

$$
L\left(s, \chi, K_{d} \mid K\right)=L(s, \tilde{\chi}) .
$$

Furthermore, since $K \subseteq K_{\chi} \subseteq K_{d}$, we see by $[9,5.1 .5]$ and the last paragraph of $[9, \S 6]$ that the conductor $\mathfrak{f}_{\chi}$ divides $(d)$; thus, $d_{\tilde{\chi}} \leqslant Q$.

Using the remarks above we conclude that $\zeta_{d}(s)$ vanishes in $\Omega(T, U)$ if and only if $L\left(s, \chi_{*}\right)$ is a factor of $\zeta_{d}(s)$ and $L\left(\beta_{*}, \chi_{*}\right)=0$. In this case, we know that $\mathfrak{f}_{\chi_{*}} \mid(d)$ and $\mathfrak{f}_{\chi_{*}} \neq 1$; thus, we can take $\mathfrak{f}=\mathfrak{f}_{\chi_{*}}$.

Lemma 3.2 There are constants $c_{3}, c_{4}, c_{5}>0$ depending only on $K$ with the property that for all $d \in \mathbb{N}_{K}, T \geqslant c_{3} d$, and $\sigma \geqslant 1-1 / c_{5}$, the number $N_{d}(\sigma, T)$ of zeros $\beta+i \gamma$ of $\zeta_{d}(s)$ with $\beta \geqslant \sigma$ and $|\gamma| \leqslant T$ satisfies the bound

$$
N_{d}(\sigma, T) \leqslant c_{4}(T d)^{c_{5}(1-\sigma)} .
$$

Proof We continue to use notation of [16, $\S 1]$. As in the proof of Lemma 3.1, for each $d \in \mathbb{N}_{K}$ let $H$ denote the trivial subgroup of the ideal class group $I((d)) / P_{(d)}$ modulo $(d)$, and note that the quantities $h_{H}$ and $d(H)$ defined by [16, Equation (1.1b)] satisfy the bound

$$
\max \left\{h_{H}, d(H)\right\} \leqslant(c d)^{n_{K}}
$$

for some constant $c=c(K)$ in view of [16, Lemma 1.16]. The result now follows by applying [16, Corollary 4.4] with $Q=(c d)^{n_{K}}$ and $T \geqslant c_{3} d$, where $c_{3}=c_{3}(K)$ is any constant that is large enough so that the conditions $T \gg 1$ and $T \geqslant n_{K}^{2} h_{H}^{1 / n_{K}}$ of $\left[16\right.$, Corollary 4.4] are met (for the latter condition, any number $c_{3} \geqslant c n_{K}^{2}$ suffices by the inequality above).

\section{Chebotarev Density Theorem}

Our goal in this section is to provide a lower bound for the counting function of the set

$$
\mathscr{P}_{C_{d}}=\left\{p \in \mathscr{P}_{C}: p \equiv 1(\bmod d)\right\}
$$

using an effective version of the Chebotarev density theorem given in [10].

By [13, Ch.1, Corollary 10.4] we see that $p \equiv 1(\bmod d)$ if and only if $p$ splits completely in $\mathbb{Q}_{d}$ if and only if $\left(p, \mathbb{Q}_{d} \mid \mathbb{Q}\right)=\left\{\mathbf{1}_{d}\right\}$ for $p \in \mathbb{N}_{K}$, where $\mathbf{1}_{d}$ denotes the identity element of $\operatorname{Gal}\left(\mathbb{Q}_{d} \mid \mathbb{Q}\right)$. It follows by the isomorphism in Lemma 2.1 that 
there exists a conjugacy class $C_{d}$ in $\operatorname{Gal}\left(K_{d} / \mathbb{Q}\right)$ (one that corresponds to $C \times\left\{\mathbf{1}_{d}\right\}$ ) with the property that

$$
p \in \mathscr{P}_{C_{d}} \Longleftrightarrow\left(p, K_{d} \mid \mathbb{Q}\right)=C_{d} \quad\left(p \in \mathbb{N}_{K}\right) .
$$

Accordingly, we study the function

$$
\pi_{C}(x ; d, 1)=\#\left\{p \leqslant x: p \in \mathbb{N}_{K},\left(p, K_{d} \mid \mathbb{Q}\right)=C_{d}\right\}
$$

and its weighted version

$$
\psi_{C}(x ; d, 1)=\sum_{\substack{p, m: p^{m} \leqslant x \\\left(p^{n}, K_{d} \mid \mathbb{Q}\right)=C_{d}}} \log p,
$$

where the sum is taken over primes in $\mathbb{N}_{K}$. Our main result is the following:

Theorem 4.1 There are constants $x_{1}, B>0$ depending only on $K$ with the property that for all $x \geqslant x_{1}$ and every $d \in \mathbb{N}_{K}$ with $d \leqslant x^{B}$,

$$
\pi_{C}(y ; d, 1) \geqslant \frac{|C|}{2 n_{K} \phi(d)} \frac{y}{\log y} \quad\left(x^{4 / 5} \leqslant y \leqslant x\right)
$$

whenever $\zeta_{d}(s)$ has no zeros in the region

$$
\Omega_{B}(x)=\left\{\beta+i \gamma: \beta \geqslant 1-\frac{c_{1}}{\log \left(c_{2} x^{4 B}\right)},|\gamma| \leqslant x^{3 B}\right\} .
$$

Proof Let $B=B(K)$ be a constant in the interval $\left(0, \frac{1}{100}\right)$ to be further determined below. For convenience, we set

$$
\theta_{B}(y)=\frac{c_{1} \log y}{\log \left(c_{2} y^{5 B}\right)}
$$

For $x^{4 / 5} \leqslant y \leqslant x$, we have

$$
1-\frac{\theta_{B}(y)}{\log y} \geqslant 1-\frac{c_{1}}{\log \left(c_{2} x^{4 B}\right)} \quad \text { and } \quad y^{3 B} \leqslant x^{3 B}
$$

hence the region

$$
\widetilde{\Omega}_{B}(y)=\left\{\beta+i \gamma: \beta \geqslant 1-\frac{\theta_{B}(y)}{\log y},|\gamma| \leqslant y^{3 B}\right\}
$$

is contained in $\Omega_{B}(x)$; therefore, $\zeta_{d}(s)$ has no zeros in $\widetilde{\Omega}_{B}(y)$ whenever it has no zeros in $\Omega_{B}(x)$.

Let $g$ be a fixed element of $C_{d}$ with $d \in \mathbb{N}_{K}$ and $d \leqslant x^{B}, H=\langle g\rangle$ the cyclic subgroup of $G$ generated by $g, E$ the fixed field of $H$, and $\widehat{H}$ the dual of $H$, i.e., the set of irreducible characters $\chi: H \rightarrow \mathbb{C}^{\times}$. 
Applying [10, Theorem 7.1] with the choices $G=\operatorname{Gal} K_{d} \mid \mathbb{Q}$ and $T=y^{3 B}$, and taking into account the bounds

$$
\begin{aligned}
& |G|=n_{K_{d}}=\phi(d) n_{K} \underset{K}{\ll} d \leqslant y^{2 B} \\
& \log \left|\mathscr{D}_{K_{d}}\right| \ll \phi(d)\left(\log \left|\mathscr{D}_{K}\right|+n_{K} \log d\right) \underset{K}{\ll} y^{2 B} \log y,
\end{aligned}
$$

which hold by Lemma 2.1 for all $d \leqslant x^{B} \leqslant y^{5 B / 4}$, we derive that

$$
\psi_{C}(y ; d, 1)-\frac{|C|}{|G|} y+\frac{|C|}{|G|} Z_{B}(y) \underset{K}{\ll} \frac{|C|}{|G|} y^{1-B} \log y,
$$

where we have used $\left|C_{d}\right|=|C|$, and

$$
Z_{B}(y)=\sum_{\chi \in \widehat{H}} \bar{\chi}(g)\left(\sum_{\substack{\rho \\|\gamma| \leqslant T}} \frac{y^{\rho}}{\rho}-\sum_{\substack{\rho \\|\rho|<\frac{1}{2}}} \frac{1}{\rho}\right) .
$$

Here, the inner sums are taken over the nontrivial zeros $\rho=\beta+i \gamma$ of the Artin $L$-functions $L\left(s, \chi, K_{d} \mid E\right)$ that satisfy

$$
\zeta_{d}(s)=\prod_{\chi \in \widehat{H}} L\left(s, \chi, K_{d} \mid E\right)
$$

Assuming that $\zeta_{d}(s)$ has no zeros in the region $\Omega_{B}(x)$, it follows by the functional equation of $\zeta_{d}(s)$ that every zero $\rho=\beta+i \gamma$ of $\zeta_{d}(s)$, and thus also of each $L\left(s, \chi, K_{d} \mid E\right)$, lies outside of the region

$$
\left\{\beta+i \gamma: 0 \leqslant \beta \leqslant \frac{\theta_{B}(y)}{\log y},|\gamma| \leqslant y^{3 B}\right\}
$$

and thus $|\rho|>\theta_{B}(y) / \log y \underset{K}{\gg} 1 / \log y$ for every such zero. We conclude that

$$
\sum_{\substack{\rho: \beta<\frac{1}{2} \\|\gamma| \leqslant 1}} \frac{y^{\rho}}{\rho}-\sum_{\substack{\rho \\|\rho|<\frac{1}{2}}} \frac{1}{\rho} \ll y^{1 / 2} \sum_{\substack{\rho \\|\gamma| \leqslant 1}} \frac{1}{|\rho|} \underset{K}{\ll} n_{\chi}(0) y^{1 / 2} \log y,
$$

where $n_{\chi}(t)$ is the number of zeros $\beta+i \gamma$ of $L\left(s, \chi, K_{d} \mid E\right)$ such that $0<\beta<1$ and $|\gamma-t| \leqslant 1$. By [10, Lemma 5.4],

$$
n_{\chi}(t) \ll \log d_{\chi}+\frac{n_{K} \phi(d)}{|H|} \log (|t|+2),
$$


where $d_{\chi}=\left|\mathscr{D}_{E}\right| \mathrm{N}_{E / \mathbb{Q}}\left(\mathfrak{f}_{\chi}\right)$. Summing over all characters $\chi \in \widehat{H}$ and using (4.5) we see that

$$
\begin{aligned}
& \sum_{\chi \in \widehat{H}} \bar{\chi}(g)\left(\sum_{\substack{\rho: \beta<\frac{1}{2} \\
|\gamma| \leqslant 1}} \frac{y^{\rho}}{\rho}-\sum_{\substack{\rho \\
|\rho|<\frac{1}{2}}} \frac{1}{\rho}\right) \\
& \quad \underset{K}{\ll} y^{1 / 2} \log y \sum_{\chi \in \widehat{H}}\left(\log d_{\chi}+\frac{d}{|H|}\right)=y^{1 / 2} \log y\left(\log \left|\mathscr{D}_{K_{d}}\right|+y^{2 B}\right) \\
& \underset{K}{\ll} y^{1 / 2+2 B} \log ^{2} y,
\end{aligned}
$$

where the equaliy $\left|\mathscr{D}_{K_{d}}\right|=\prod_{\chi} d_{\chi}$ follows from [13, Ch. 3, Corollary 2.10] and the Conductor-Discriminant formula [13, Ch.7, Proposition 11.9]. Moreover,

$$
\sum_{\substack{\rho: \beta<\frac{1}{2} \\ 1<|\gamma| \leqslant y^{3 B}}} \frac{y^{\rho}}{\rho} \ll y^{1 / 2} \sum_{\substack{\rho \\ 1 \leqslant|\gamma| \leqslant y^{3 B}}} \frac{1}{|\rho|} \leqslant y^{1 / 2} \sum_{j=1}^{\left\lfloor y^{3 B}\right\rfloor} \sum_{\substack{\rho \\ j \leqslant|\gamma| \leqslant j+1}} \frac{1}{|\rho|} ;
$$

thus, summing over the characters we obtain

$$
\begin{aligned}
\sum_{\chi \in \widehat{H}} \bar{\chi}(g) \sum_{\substack{\rho: \beta<\frac{1}{2} \\
1<|\gamma| \leqslant y^{3 B}}} \frac{y^{\rho}}{\rho} & \ll y^{1 / 2} \sum_{j=1}^{\left\lfloor y^{3 B}\right\rfloor} \frac{1}{j} \sum_{\chi \in \widehat{H}}\left(\log d_{\chi}+\frac{n_{K} \phi(d)}{|H|} \log (j+1)\right) \\
& \ll y^{1 / 2+2 B} \log ^{2} y .
\end{aligned}
$$

In view of (4.6) and (4.7) we have

$$
Z_{B}(y)=\sum_{\chi \in \widehat{H}} \bar{\chi}(g) \sum_{\substack{\rho: \beta \geqslant \frac{1}{2} \\|\gamma| \leqslant y^{3 B}}} \frac{y^{\rho}}{\rho}+O_{K}\left(y^{1 / 2+2 B} \log ^{2} y\right) .
$$

To estimate the sum in (4.8), we use ideas (and notation) from the proof of [1, Theorem 2.1]. For each zero $\rho=\beta+i \gamma$ in the sum, we have $\left|y^{\rho}\right|=y^{\beta}$ and $|\rho| \geqslant \frac{1}{4}+|\gamma| \gg 1+|\gamma|$. Fix $\chi \in \widehat{H}$ and write $\sum_{\sigma}^{\alpha}$ for any sum over all zeros $\beta+i \gamma$ of $L\left(s, \chi, K_{d} / E\right)$ with $\sigma \leqslant \beta<\alpha$ and $|\gamma| \leqslant y^{3 B}$. Put $\tau=1-\theta_{B}(y) / \log y$, and note that $\sum_{\tau}^{1} \frac{y^{\rho}}{\rho}=0$, since $\zeta_{d}(s)$ has no zeros in $\widetilde{\Omega}_{B}(y)$. Hence, using the upper bound $y^{\beta} \leqslant y^{1-1 / c_{5}}$ when $\beta \leqslant 1-1 / c_{5}$ and the identity $y^{\beta}=y^{1-1 / c_{5}}+\log y \int_{1-1 / c_{5}}^{\beta} y^{\sigma} d \sigma$ 
when $\beta$ lies in the range $1-1 / c_{5} \leqslant \beta \leqslant \tau$, it follows that

$$
\begin{aligned}
\sum_{\substack{\rho \\
\beta \geqslant \frac{1}{2},|\gamma| \leqslant y^{3 B}}} \frac{y^{\rho}}{\rho} & =\sum_{1 / 2}^{1-1 / c_{5}} \frac{y^{\rho}}{\rho}+\sum_{1-1 / c_{5}}^{\tau} \frac{y^{\rho}}{\rho} \ll \sum_{1 / 2}^{1-1 / c_{5}} \frac{y^{\beta}}{1+|\gamma|}+\sum_{1-1 / c_{5}}^{\tau} \frac{y^{\beta}}{1+|\gamma|} \\
& \ll y^{1-1 / c_{5}} \sum_{1 / 2}^{\tau} \frac{1}{1+|\gamma|}+\log y \sum_{1-1 / c_{5}}^{\tau} \frac{1}{1+|\gamma|} \int_{1-1 / c_{5}}^{\beta} y^{\sigma} d \sigma \\
& \ll y^{1-1 / c_{5}} \sum_{\substack{\rho \\
|\gamma| \leqslant y^{3 B}}} \frac{1}{1+|\gamma|}+\log y \int_{1-1 / c_{5}}^{\tau} y^{\sigma}\left(\sum_{\sigma}^{\tau} \frac{1}{1+|\gamma|}\right) d \sigma
\end{aligned}
$$

Summing over all characters and using (4.5), the first term above can be bounded as before:

$$
\begin{aligned}
\sum_{\chi \in \widehat{H}} \bar{\chi}(g) \sum_{\substack{\rho \\
|\gamma| \leqslant y^{3 B}}} \frac{1}{1+|\gamma|} & \leqslant \sum_{\chi \in \widehat{H}} \sum_{j=0}^{\left\lfloor y^{3 B}\right\rfloor} \sum_{\substack{\rho \\
j \leqslant|\gamma| \leqslant j+1}} \frac{1}{1+|\gamma|} \\
& \ll \sum_{j=0}^{\left\lfloor y^{3 B}\right\rfloor} \frac{d \log d+d \log (1+j)}{j+1} \ll y^{2 B} \log ^{2} y .
\end{aligned}
$$

Let $N_{\chi}(\sigma, T)$ be the number of zeros $\beta+i \gamma$ of $L\left(s, \chi, K_{d} \mid E\right)$ with $\beta \geqslant \sigma$ and $|\gamma| \leqslant T$. Then, it follows by partial summation that for $\sigma \geqslant 1-1 / c_{5}$,

$$
\sum_{\sigma}^{\tau} \frac{1}{1+|\gamma|} \leqslant N_{\chi}\left(\sigma, c_{3} d\right)+\frac{N_{\chi}\left(\sigma, y^{3 B}\right)}{y^{3 B}}+\int_{c_{3} d}^{y^{3 B}} \frac{N_{\chi}(\sigma, t)}{t^{2}} d t .
$$

Summing over all characters $\chi$ once again we obtain

$$
\sum_{\chi \in \widehat{H}} \bar{\chi}(g) \sum_{\sigma}^{\tau} \frac{1}{1+|\gamma|} \ll N_{d}\left(\sigma, c_{3} d\right)+\frac{N_{d}\left(\sigma, y^{3 B}\right)}{y^{3 B}}+\int_{c_{3} d}^{y^{3 B}} \frac{N_{d}(\sigma, t)}{t^{2}} d t .
$$

By Lemma 3.2, we have for all $\sigma \geqslant 1-1 / c_{5}$ and $d \leqslant x^{B} \leqslant y^{2 B}$,

$$
\begin{aligned}
\sum_{\chi \in \widehat{H}} \bar{\chi}(g) \sum_{\sigma}^{\tau} \frac{1}{1+|\gamma|} & \ll c_{4}\left(c_{3} d^{2}\right)^{c_{5}(1-\sigma)}+\frac{c_{4}\left(y^{3 B} d\right)^{c_{5}(1-\sigma)}}{y^{3 B}}+\int_{c_{3} d}^{y^{3 B}} \frac{c_{4}(t d)^{c_{5}(1-\sigma)}}{t^{2}} d t \\
& \ll y^{4 c_{5} B(1-\sigma)}+y^{2 c_{5} B(1-\sigma)} \int_{1}^{y^{3 B}} \frac{t^{c_{5}(1-\sigma)}}{t^{2}} d t .
\end{aligned}
$$


Using the bound

$$
\int_{1}^{y^{3 B}} \frac{t^{c_{5}(1-\sigma)}}{t^{2}} d t \underset{K}{\ll} \begin{cases}\log y & \text { if } 1-1 / c_{5} \leqslant \sigma \leqslant 1-1 /\left(2 c_{5}\right), \\ 1 & \text { if } \sigma \geqslant 1-1 /\left(2 c_{5}\right),\end{cases}
$$

and assuming that $B<1 /\left(4 c_{5}\right)$, we derive that

$$
\begin{aligned}
\int_{1-1 / c_{5}}^{\tau} & y^{\sigma}\left(\sum_{\chi \in \widehat{H}} \bar{\chi}(g) \sum_{\sigma}^{\tau} \frac{1}{1+|\gamma|}\right) d \sigma \\
& \ll \int_{1-1 / c_{5}}^{\tau} y^{\sigma} \cdot y^{4 c_{5} B(1-\sigma)} d \sigma+\int_{1-1 / c_{5}}^{1-1 /\left(2 c_{5}\right)} y^{\sigma} \cdot y^{2 c_{5} B(1-\sigma)} \log y d \sigma \\
= & y^{4 c_{5} B} \int_{1-1 / c_{5}}^{\tau} y^{\sigma\left(1-4 c_{5} B\right)} d \sigma+y^{2 c_{5} B} \log y \int_{1-1 / c_{5}}^{1-1 /\left(2 c_{5}\right)} y^{\sigma\left(1-2 c_{5} B\right)} d \sigma \\
& \ll y^{4 c_{5} B} \frac{y^{\tau\left(1-4 c_{5} B\right)}}{\left(1-4 c_{5} B\right) \log y}+y^{2 c_{5} B} \frac{y^{\left(1-1 /\left(2 c_{5}\right)\right)\left(1-2 c_{5} B\right)}}{\left(1-2 c_{5} B\right)} \\
= & \frac{y \exp \left(-\left(1-4 c_{5} B\right) \theta_{B}(y)\right)}{\left(1-4 c_{5} B\right) \log y}+\frac{y^{1+B-1 /\left(2 c_{5}\right)}}{\left(1-2 c_{5} B\right)},
\end{aligned}
$$

where we have used the definition of $\tau$ in the last step. Combining this bound with (4.8), (4.9), and (4.10) and assuming further that $B \leqslant 1 /\left(5 c_{5}\right)$, we find that

$$
Z_{B}(y) \underset{K}{\ll} y \exp \left(-\frac{1}{5} \theta_{B}(y)\right)
$$

Finally, using (4.4) we see that

$$
\left|\psi_{C}(y ; d, 1)-\frac{|C|}{|G|} y\right| \leqslant \frac{|C|}{|G|} c y\left(\exp \left(-\frac{1}{5} \theta_{B}(y)\right)+y^{-B} \log ^{2} y\right)
$$

for some sufficiently large constant $c=c(K)$.

To finish the proof, we now put

$$
B=\min \left\{\frac{1}{100}, \frac{1}{5 c_{5}}, \frac{c_{1}}{30 \log (6 c)}\right\}
$$

Note that $B$ depends only on $K$, the bound (4.11) holds, and we have

$$
c \exp \left(-\frac{c_{1}}{30 B}\right) \leqslant \frac{1}{6}
$$

On the other hand, from the definition (4.3) one sees that $\theta_{B}(y) \geqslant c_{1} /(6 B)$ holds for any $y \geqslant y_{1}$, where $y_{1}=\exp \left(\left(\log c_{2}\right) / B\right)$. Therefore,

$$
c \exp \left(-\frac{1}{5} \theta_{B}(y)\right) \leqslant \frac{1}{6} \quad\left(y \geqslant y_{1}\right)
$$


Increasing the value of $y_{1}$ if necessary, we also have

$$
c y^{-B} \log ^{2} y \leqslant \frac{1}{6} \quad\left(y \geqslant y_{1}\right) .
$$

Put $x_{1}=y_{1}^{5 / 4}$, so that the condition $y \geqslant y_{1}$ is satisfied whenever $x^{4 / 5} \leqslant y \leqslant x$ and $x \geqslant x_{1}$. Combining the bounds (4.11), (4.12), and (4.13) we obtain

$$
\psi_{C}(y ; d, 1) \geqslant \frac{2|C|}{3|G|} y \quad\left(x^{4 / 5} \leqslant y \leqslant x\right)
$$

for all $x \geqslant x_{1}$. Partial summation yields

$$
\pi_{C}(y ; d, 1) \geqslant \frac{2|C|}{3|G|} \frac{y}{\log y}-\frac{4 \sqrt{y}}{\log y} \geqslant \frac{|C|}{2|G|} \frac{y}{\log y} \quad\left(x^{4 / 5} \leqslant y \leqslant x\right),
$$

where the last inequality holds when $\sqrt{y} \geqslant 24|G| /|C|$, which is guaranteed by our choice of $B$ and $d$ with $d \leqslant x^{B}$. We finish the proof by noting that $|G|=n_{K} \phi(d)$.

\section{Construction of Carmichael Numbers}

In view of Theorem 4.1, our construction of Carmichael numbers with the property stated in Theorem 1.1 follows closely that given in [1]. We shall be brief, since most of the details are the same. Our principal tool is the following variant of [1, Theorem 3.1].

Lemma 5.1 Let the constants $x_{1}, B$ have the property stated in Theorem 4.1, and suppose that $x \geqslant x_{1}$. If $L$ is any squarefree number in $\mathbb{N}_{K}$ that is not divisible by any prime exceeding $x^{(1-B) / 2}$ and

$$
\sum_{\text {prime } q \mid L} \frac{1}{q} \leqslant \frac{1}{60 n_{K}},
$$

then there is a positive number $k \leqslant x^{1-B}$ with $\operatorname{gcd}(k, L)=1$ such that

$$
\#\left\{d \mid L: d k+1 \in \mathscr{P}_{C}, d k+1 \leqslant x\right\} \geqslant \frac{1}{6 n_{K} \log x} \cdot \#\left\{d \mid L: d \leqslant x^{B}\right\} .
$$

Proof We use ideas (and notation) from the proof of [1, Theorem 3.1].

Observe that the region $\Omega_{B}(x)$ defined by (4.2) is the same as the region $\Omega(T, U)$ defined by (3.1) when we put $T=x^{3 B}$ and $U=x^{B}$.

Fix a prime $p_{0}$ with the property that $p_{0} \mid \mathrm{N}_{K / \mathbb{Q}}(\mathfrak{f})$, where $\mathfrak{f}=\mathfrak{f}\left(K, x^{B}, x^{3 B}\right)$ is given by Lemma 3.1. If $L$ is divisible by $p_{0}$ let $L^{\prime}=L / p_{0}$; otherwise, let $L^{\prime}=L$. Note that

$$
\#\left\{d \mid L^{\prime}: d \leqslant y\right\} \geq \frac{1}{2} \cdot \#\{d \mid L: d \leqslant y\} \quad(y \geqslant 1)
$$


(see [1, p. 716]). Since $p_{0} \nmid L^{\prime}$, for every divisor $d$ of $L^{\prime}$ with $d \leqslant x^{B}$, Lemma 3.1 shows that $\zeta_{d}(s)$ has no zeros in $\Omega_{B}(x)$; therefore, using the lower bound (4.1) from Theorem 4.1 we have

$$
\pi_{C}\left(d x^{1-B} ; d, 1\right) \geqslant \frac{|C|}{2 n_{K}} \frac{d x^{1-B}}{\phi(d) \log x} .
$$

On the other hand, since any prime divisor $q$ of $L$ does not exceed $x^{(1-B) / 2}$, we have from [12, Theorem 2],

$$
\pi_{C}\left(d x^{1-B} ; d q, 1\right) \leqslant \pi\left(d x^{1-B} ; d q, 1\right) \leqslant \frac{10}{q} \frac{d x^{1-B}}{\phi(d) \log x} .
$$

Therefore, the number of primes $p \in \mathscr{P}_{C_{d}}$ with $p \leqslant d x^{1-B}$ and $\operatorname{gcd}((p-1) / d, L)=1$ is at least

$$
\begin{aligned}
& \pi_{C}\left(d x^{1-B} ; d, 1\right)-\sum_{\text {prime } q \mid L} \pi_{C}\left(d x^{1-B} ; d q, 1\right) \\
& \geqslant\left(\frac{1}{2 n_{K}}-10 \sum_{\text {prime } q \mid L} \frac{1}{q}\right) \frac{d x^{1-B}}{\phi(d) \log x} \geqslant \frac{x^{1-B}}{3 n_{K} \log x} .
\end{aligned}
$$

Using this bound together with (5.1) (instead of [1, Equation (3.1)]), the proof can be concluded in the same manner as that of [1, Theorem 3.1]; the remaining details are omitted.

We are now in a position to establish a quantitative version of Theorem 1.1.

Theorem 5.2 There are constants $x_{0}, c_{0}>0$ depending only on $K$ such that for all $x \geqslant x_{0}$, there are at least $x^{c_{0}}$ Carmichael numbers up to $x$ that are composed solely of primes that split completely in $K$.

Proof To prove this, we only need to modify the proof of [1, Theorem 4.1] slightly, as follows.

Let $\mathcal{E}$ be the set of numbers $E \in(0,1)$ for which there exists a constant $x_{2}>0$ depending only on $E$ such that

$$
\pi\left(x, x^{1-E}\right) \underset{E}{\gg} \pi(x) \quad\left(x \geqslant x_{2}\right),
$$

where $\pi(x, y)$ denotes the number of primes $p \leqslant x$ such that $p-1$ is free of prime factors exceeding $y$.

Fix $E=3 / 5$, which lies in the set $\mathcal{E}$ (see, e.g., [7]), and let $x_{2}$ be a number for which the bound (5.2) holds. Let $x_{1}, B$ be numbers with the property stated in Theorem 4.1, and put $x_{3}=\max \left\{x_{1}, x_{2}\right\}$. Note that our choice of $x_{3}$ depends only on $K$.

Let $y \geq 2$ be a parameter and $Q$ the set of primes $q \in \mathbb{N}_{K}$ with

$$
y^{5 / 2} / \log y<q \leqslant y^{5 / 2}
$$


for which $q-1$ is free of prime factors exceeding $y$. By (5.2)

$$
|Q| \geqslant \pi\left(y^{5 / 2}, y\right)-\pi\left(y^{5 / 2} / \log y\right)-\sum_{q \mid \mathscr{D}_{K}} 1 \gg y^{5 / 2} / \log y
$$

for all sufficiently large $y$. Let $L$ be the product of primes in $Q$; then

$$
\log L=\sum_{q \in Q} \log q \leqslant \sum_{q \leqslant y^{5 / 2}} \log q=\vartheta\left(y^{5 / 2}\right) \leqslant 1.1 y^{5 / 2}
$$

for all $y>0$, where we have used [14] for the last inequality. Furthermore,

$$
\lambda(L)=\prod_{p^{a}|| \lambda(L)} p^{a} \leqslant \prod_{p \leqslant y} p^{\left\lfloor\frac{\log 5 / 2}{\log p}\right\rfloor} \leqslant y^{5 \pi(y) / 2} \leqslant e^{\pi \cdot y},
$$

where the last inequality follows again by [14]. We also have

$$
n\left(G_{L}\right) \leqslant \lambda(L)(1+\log L) \leqslant e^{\pi y}\left(1+1.1 y^{5 / 2}\right) \leqslant e^{5 y},
$$

where $G_{L}=(\mathbb{Z} / L \mathbb{Z})^{*}$.

Let $x=e^{y^{1+\delta}}$, where $\delta=5 \varepsilon /(8 B)$. Since

$$
\sum_{\text {prime } q \mid L} \frac{1}{q} \leqslant \sum_{y^{5 / 2} / \log y<q \leqslant y^{5 / 2}} \frac{1}{q} \leqslant 4 \frac{\log \log y}{5 \log y} \leqslant \frac{1}{60 n_{K}}
$$

for sufficiently large $y$, it follows from Lemma 5.1 that there exists an integer $k$ coprime to $L$, for which the set $\mathcal{P}$ of primes $p \leqslant x$ with $p \in \mathscr{P}_{C}$ and $p=d k+1$ for some divisor $d$ of $L$, satisfies

$$
|\mathcal{P}| \geqslant \frac{1}{6 n_{K} \log x} \cdot \#\left\{d \mid L: 1 \leqslant d \leqslant x^{B}\right\} .
$$

The product of any

$$
u:=\left[\frac{\log \left(x^{B}\right)}{\log y^{5 / 2}}\right]
$$

distinct prime factors of $L$, is a divisor $d$ of $L$ with $d \leqslant x^{B}$. We deduce from (5.3) that

$$
\#\left\{d \mid L: 1 \leqslant d \leqslant x^{B}\right\} \geq\left(\begin{array}{c}
\omega(L) \\
u
\end{array}\right) \geq\left(\frac{\omega(L)}{u}\right)^{u} \geqslant\left(\frac{c y^{5 / 2}}{2 B \log x}\right)^{u} .
$$

Thus, by (5.5) and the identity $(5 / 2-1-\delta) 2 B / 5=3 B / 5-\varepsilon / 4$,

$$
|\mathcal{P}| \geqslant \frac{1}{6 n_{K} \log x}\left(\frac{c}{2 B} y^{5 / 2-1-\delta}\right)^{\left\lfloor\frac{2 B \log x}{5 \log y}\right\rfloor} \geqslant x^{3 B / 5-\varepsilon / 3}
$$


for all sufficiently large values of $y$. Now take $\mathcal{P}^{\prime}=\mathcal{P} \backslash Q$. Since $|Q| \leqslant y^{5 / 2}$, it follows by the above inequality that

$$
\left|\mathcal{P}^{\prime}\right| \geqslant x^{3 B / 5-\varepsilon / 2}
$$

for all sufficiently large values of $y$.

We may view $\mathcal{P}^{\prime}$ as a subset of the group $(\mathbb{Z} / L \mathbb{Z})^{\star}$ by considering the residue class of each $p \in \mathcal{P}^{\prime}$ modulo $L$. If $S$ is a subset of $\mathcal{P}^{\prime}$ that contains more than one element and if

$$
\Pi(S):=\prod_{p \in S} p \equiv 1(\bmod L),
$$

then $\Pi(S)$ is a Carmichael number. Indeed, every member of $\mathcal{P}^{\prime}$ is $1 \bmod k$ so that $\Pi(S) \equiv 1(\bmod k)$, and thus $\Pi(S) \equiv 1(\bmod k L)$, since $(k, L)=1$. However, if $p \in \mathcal{P}^{\prime}$, then $p \in \mathcal{P}$ so that $p-1$ divides $k L$. Thus $\Pi(S)$ satisfies Korselt's criterion.

Let $t=e^{y^{1+\delta / 2}}$. Then, by [1, Proposition 1.2], we see that the number of Carmichael numbers of the form $\Pi(S)$, where $S \subseteq \mathcal{P}^{\prime}$ and $|S| \leqslant t$, is at least

$$
\left(\begin{array}{c}
\left|\mathcal{P}^{\prime}\right| \\
\lfloor t\rfloor
\end{array}\right)\left(\begin{array}{c}
\left|\mathcal{P}^{\prime}\right| \\
n\left(G_{L}\right)
\end{array}\right)^{-1} \geqslant\left(\frac{\left|\mathcal{P}^{\prime}\right|}{\lfloor t\rfloor}\right)^{\lfloor t\rfloor}\left|\mathcal{P}^{\prime}\right|^{-n\left(G_{L}\right)} \geqslant x^{t(3 B / 5-\varepsilon)}
$$

for all sufficiently large values of $y$, using (5.4) and (5.6). But each such Carmichael number $\Pi(S)$ so formed is such that $\Pi(S) \leqslant x^{t}$. Thus for $X=x^{t}$ we have $C(X) \geqslant$ $X^{3 B / 5-\varepsilon}$ for all sufficiently large $y$. But $X=\exp \left(y^{1+\delta} \exp \left(y^{1+\delta / 2}\right)\right)$, so that $C(X) \geqslant$ $X^{3 B / 5-\varepsilon}$ for all sufficiently large values of $X$. Since $y$ can be uniquely determined from $X$, we complete the proof by taking $c_{0}=E B / 2$.

\section{References}

[1] W. R. Alford, A. Granville, and C. Pomerance, There are infinitely many Carmichael numbers. Ann. of Math. (2) 139(1994), no. 3, 703-722. http://dx.doi.org/10.2307/2118576

[2] W. D. Banks, Carmichael numbers with a square totient. Canad. Math. Bull. 52(2009), no. 1, 3-8. http://dx.doi.org/10.4153/CMB-2009-001-7

[3] W. D. Banks and C. Pomerance, On Carmichael numbers in arithmetic progressions. J. Austral. Math. Soc. 88(2010), no. 3, 313-321. http://dx.doi.org/10.1017/S1446788710000169

[4] D. A. Cox, Primes of the form $x^{2}+n y^{2}$. Fermat, class field theory and complex multiplication. John Wiley \& Sons, Inc., New York, 1989.

[5] D. S. Dummit and R. M. Foote, Abstract algebra. Third ed. John Wiley \& Sons, Inc., Hoboken, NJ, 2004.

[6] A. Ekstrom, C. Pomerance, and D. S. Thakur, Infinitude of elliptic Carmichael numbers. J. Austral. Math. Soc. 92(2012), 45-60. http://dx.doi.org/10.1017/S1446788712000080

[7] J. B. Friedlander, Shifted primes without large prime factors. In: Number theory and applications (Banff, AB, 1988), NATO Adv. Sci. Inst. Ser. C Math. Phys. Sci., 265, Kluwer Acad. Publ., Dordrecht, 1989, pp. 393-401.

[8] J. Grantham, There are infinitely many Perrin pseudoprimes. J. Number Theory 130(2010), no. 5, 1117-1128. http://dx.doi.org/10.1016/j.jnt.2009.11.008

[9] G. J. Janusz, Algebraic number fields. Pure and Applied Mathematics, 55, Academic Press, New York-London, 1973.

[10] J. C. Lagarias and A. M. Odlyzko, Effective versions of the Chebotarev density theorem. In: Algebraic number fields: $L$-functions and Galois properties (Proc. Sympos., Univ. Durham, Durham, 1975), Academic Press, London, 1977, pp. 409-464.

[11] K. Matomäki, Carmichael numbers in arithmetic progressions. J. Austral. Math. Soc., to appear. 
[12] H. L. Montgomery and R. C. Vaughan, The large sieve. Mathematika 20(1973), 119-134. http://dx.doi.org/10.1112/S0025579300004708

[13] J. Neukirch, Algebraic number theory. Grundlehren der Mathematischen Wissenschaften, 322, Springer-Verlag, Berlin, 1999.

[14] J. B. Rosser and L. Schoenfeld, Approximate formulas for some functions of prime numbers. Illinois J. Math. 6(1962), 64-94.

[15] L. C. Washington, Introduction to cyclotomic fields. Second ed., Graduate Texts in Mathematics, 83, Springer-Verlag, New York, 1997.

[16] A. Weiss, The least prime ideal. J. Reine Angew. Math. 338(1983), 56-94.

Department of Mathematics, University of Missouri, Columbia, MO 65211 USA

e-mail: bankswd@missouri.edu

Department of Mathematics, Bilkent University, 06800 Bilkent, Ankara, TURKEY

e-mail: guloglua@fen.bilkent.edu.tr

Department of Mathematics, University of Missouri, Columbia, MO 65211, USA

e-mail: amydm6@mail.missouri.edu 